\title{
Prolonged Sinus Pauses Revealing a Paroxysmal Extreme Pain Disorder: Is it a Frequent Situation?

\section{Abstract}

Title: Paroxysmal extreme pain disorder (PEPD) is an autosomal dominant painful neuropathy with many, but not all, cases linked to gain-of-function mutations in SCN9A which encodes voltage-gated sodium channel $\mathrm{Na}$. 1.7. It is a very rare condition featured by flushing of the lower half of the body and excruciating burning pain caused by any stimulus below the waist or in the perianal region. PEPD may be associated with cardiovascular instability, especially prolonged sinus pauses, and thus has anesthetic implications. Pacemaker implantation is the alternative therapeutic option, but its indications have not been clarified yet.

Background: This condition is well described in neurological literature, but to our knowledge, this is the first case report of a patient with paroxysmal extreme pain disorder with prolonged sinus pauses requiring anesthesia for an epicardial pacemaker even with the perioperative risk of the pathology. This clinical observation can help for a better management and understanding of the cardiac risk complications of PEPD especially for an infant whose diagnostic is frequently made at the stage of complication This clinical observation can put the item on the necessity of establishing recommendations for management of cardiac complications during PEPD.

Case report: We extensively searched the literature on cardiac pacing in patients with PEPD and we described a new case of a 9 month old infant who was admitted in the emergency department for an episode of malaise apnea and hemifacial cyanosis relevant to PEPD. The neurologic exploration was normal. The diagnostic was confirmed by genetic study. The 24 hours recording demonstrated long pauses of 15 seconds during the crisis justifying the implantation of epicardial pacemaker without peri-operatory complications due to the high anesthetic risk of this pathology.

\author{
Sahar Mouram", \\ Hicham Sabor², \\ Ibtissam Fellat ${ }^{1}$
}

1 Cardiology B Department, Faculty of Medicine and Pharmacy, Rabat, Morocco.

2 Cardiology Department, Military Hospital, Faculty of Medicine and Pharmacy, Rabat, Morocco.

\section{Contact information:}

\section{Sahar Mouram.}

Cardiology B Department.

Address: Faculty of Medicine and Pharmacy, Rabat, Morocco.

Tel: 00212(0)661630785.

झ mouram.sahar@gmail.com 
Conclusion: Paroxysmal extreme pain disorder is a highly distinctive sodium channelopathy with incompletely carbamazepine-sensitive bouts of pain and sympathetic nervous system dysfunction. It is most likely to be misdiagnosed as epilepsy and, particularly in infancy, as hyperekplexia and reflex anoxic seizures. Clinicians must evocate this diagnostic even any clinical suspicion given the severity of cardiac complications.

\section{Keywords}

Paroxysmal Extreme Pain

Disorder; Sinus Pauses;

Anesthetic Risk; Pacemaker.

\section{Introduction}

Paroxysmal extreme pain disorder (PEPD) is an autosomal dominant painful neuropathy with many, but not all, cases linked to gain-of-function mutations in SCN9A which encodes voltage-gated sodium channel Na. 1.7. The paper should be of interest because this clinical observation can put the item on the severity of the cardiac risk complications of this disease especially for an infant whose diagnostic is frequently made at the stage of complication. This clinical observation can help for a better understanding of the management of anesthetic process Because of the potential for cardiovascular instability

\section{Observation}

A 9-month-old infant was brought to the emergency department after an episode of malaise and hemifacial cyanosis. Her parent reported that the crisis was triggered by crying. The infant had vegetative manifestations like hemifacial redness, snorkeling and sweats with foam at the lips, motor manifestations like rubbing feet with reduced consciousness. So the infant turned blue around the lips and began gasping for air. During this time, her eyes "rolled back", and she did not interact with her parents. There were no clonic movements, and she did not respond to physical stimulation. On arrival, she was alert and looked well. There were no focal findings on examina- tion, her development was appropriate, and her laboratory studies were normal including a blood glucose level of $5.4 \mathrm{mmol} / \mathrm{L}$. The girl's parents reported similar, less profound episodes occurring during crying since the age of 2 months. They noted that, initially, her head would go back, her eyes would roll upwards, and she would become floppy and limp. These episodes occurred only during crying and lasted a few seconds, followed by a period of sleepiness. Detailed neurological exam was normal. She was admitted to the pediatric unit for investigation.

Between these episodes, she had been well, and she did not have any evidence of intercurrent illness. She had normal attainment of developmental milestones, and there was no significant past medical history. She had been born at term with no neonatal problems after an uneventful pregnancy. She was up-to-date with the vaccination schedule, and she had not received any vaccinations recently. She was an only child, and her parents were well with no family history of seizures or epilepsy.

Electrocardiogram demonstrated sinus rhythm with a normal QT interval. Provoking cries while her vital signs were monitored provoked hypertonia, bradycardia, and brief apnea with associated desaturation. She stared straight ahead and made mouthing movements. She was calmed and given high-flow oxygen. A further similar episode occurred later while she was sleeping, this time without provocation. Follow-up included a brain 
MRI and EEG and were normal. Echocardiography was without anomalies. The 24 hours rhythm recording demonstrated the presence of long pauses up to 15 seconds (Figure 1 and 2) during the attacks thus retaining the indication of a definitive stimulation by an epicardial single chamber pacemaker VVI (St Jude medical Microny II SR, set at a back up rate of 80 beats per minute).That was implanted without difficulty during anesthesia even the high anesthetic risk of the pathology. Since the day of PM implantation and activation, the infant has had no more event.

The genetic study showed the presence of mutation in the SCN 9A confirming the diagnosis of PEPD.

\section{Discussion}

Inherited mutations in voltage-gated sodium channels (VGSCs; or Nav ) cause many disorders of excitability, including epilepsy, chronic pain, myotonia, and cardiac arrhythmias. Understanding the functional consequences of the disease-causing mutations is likely to provide invaluable insight into the roles that VGSCs play in normal and abnormal excitability.

Abnormal pain sensitivity associated with inherited and acquired pain disorders occurs through increased excitability of peripheral sensory neurons in part due to changes in the properties of voltage-gated sodium channels (Nav s) [1]. Resurgent sodium currents (INaR) are atypical currents believed to be associated with increased excitability of neurons and may have implications in pain. Mutations in Nav 1.7 (peripheral Nav isoform) associated with two genetic pain disorders, inherited erythromelalgia (IEM) and paroxysmal extreme pain disorder (PEPD), enhance Nav 1.7 function via distinct mechanisms) [1]. The universe of painful Na-channelopathies - human disorders caused by mutations in voltage-gated sodium channels - has recently expanded in three dimensions. We now know that mutations of sodium channels cause not only rare genetic 'model disorders' such as inherited erythromelalgia and channelopathy-associated insensitivity to pain but also common painful neuropathies.

The discovery of genetic variants that substantially alter an individual's perception of pain has led to a step-change in our understanding of molecular events underlying the detection and transmission of noxious stimuli by the peripheral nervous system [2]. The voltage-gated sodium ion channel Nav 1.7 is expressed selectively in sensory and autonomic neurons; inactivating mutations in SCN9A, which encodes Nav 1.7, result in congenital insensitivity to pain, whereas gain-of-function mutations in this gene produce distinct pain syndromes.

Changes in Nav 1.7 function due to mutations associated with PEPD, but not IEM, are important in INaR generation, suggesting that INaR may play a role in pain associated with PEPD [1]. This knowledge provides us with a better understanding of the mechanism of INaR generation and may lead to the development of specialized treatment for pain disorders associated with INaR. Genetic analysis of the SCN9A gene has become an important diagnostic test in the characterization of pain syndromes. Although well documented, the correlation between SCN9A genotypes and clinical phenotypes is still unclear.

Electrophysiological characterization showed that this mutation did not affect channel activation but instead resulted in incomplete fast inactivation and a small hyperpolarizing shift in steady-state slow inactivation, characteristics more commonly associated with PEPD [3]. Functional analysis of a number of mutations associated with paroxysmal extreme pain disorder has shown them to impair fast-inactivation without altering channel activation, leading to persistent current, prolonged action potentials and repetitive neuron firing in response to provoking stimuli, such as stretching and exposure to cold temperatures [2]. In contrast to IE, the SCN9A 
Figure 1: The 24 hours rhythm recording demonstrated the presence of long pauses up to 15 seconds during crying.

\begin{tabular}{|lll|}
\hline Identification utilisateur & & 06-05-2015 9:56:40 \\
\hline Nom du patient : & No. du patient $\quad:$ & \\
\hline
\end{tabular}

Sélectionné :
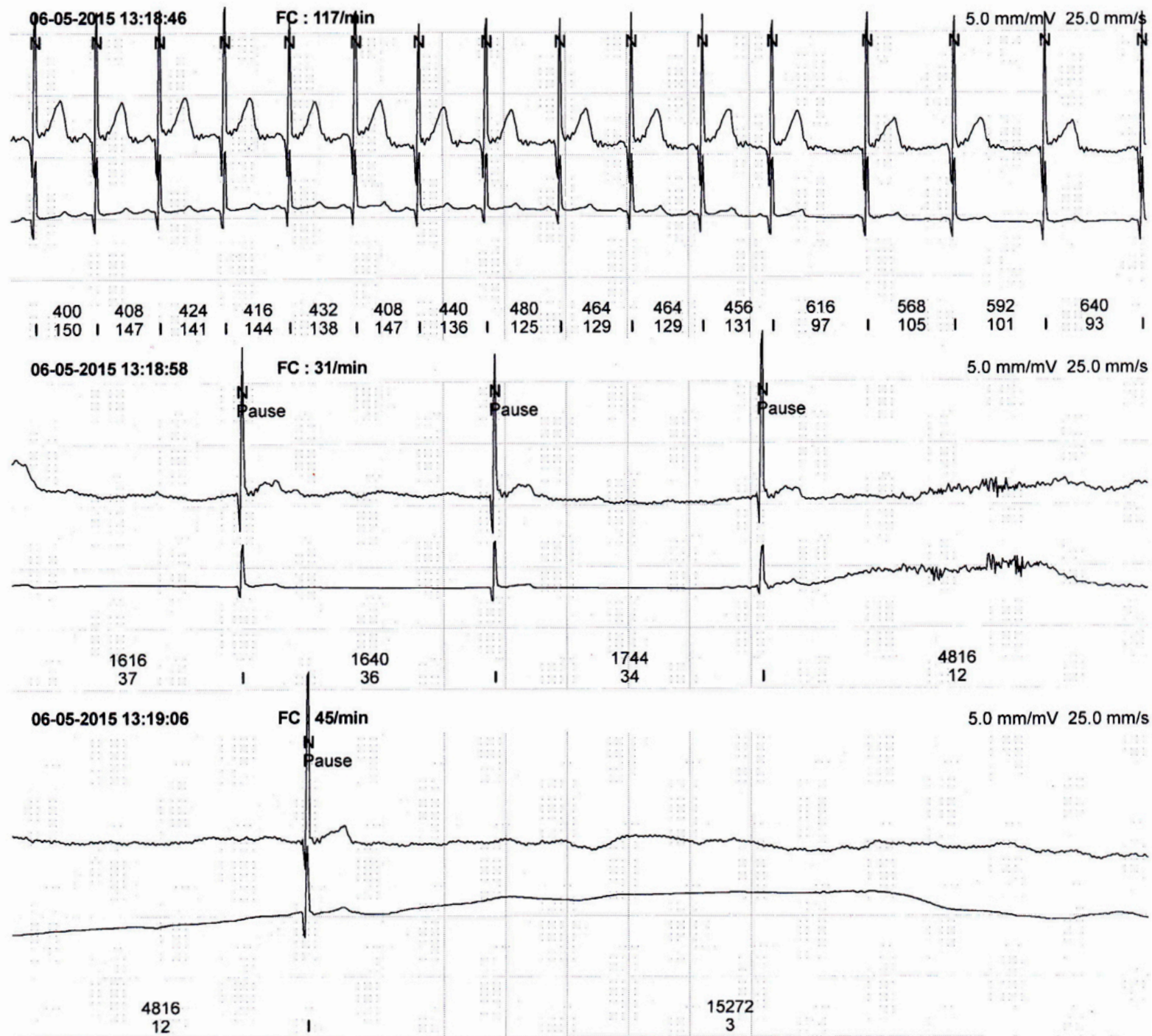

06-05-2015 13:19:10 FC: $45 / \mathrm{min}$

$5.0 \mathrm{~mm} / \mathrm{mV} 25.0 \mathrm{~mm} / \mathrm{s}$

15272 
Figure 2: The 24 hours rhythm recording demonstrated the presence of long pauses up to 15 seconds during crying.

\begin{tabular}{|lll|}
\hline Identification utilisateur & 06-05-2015 9:56:40 \\
\hline Nom du patient : & No. du patient $\quad:$ & \\
\hline
\end{tabular}

Sélectionné :

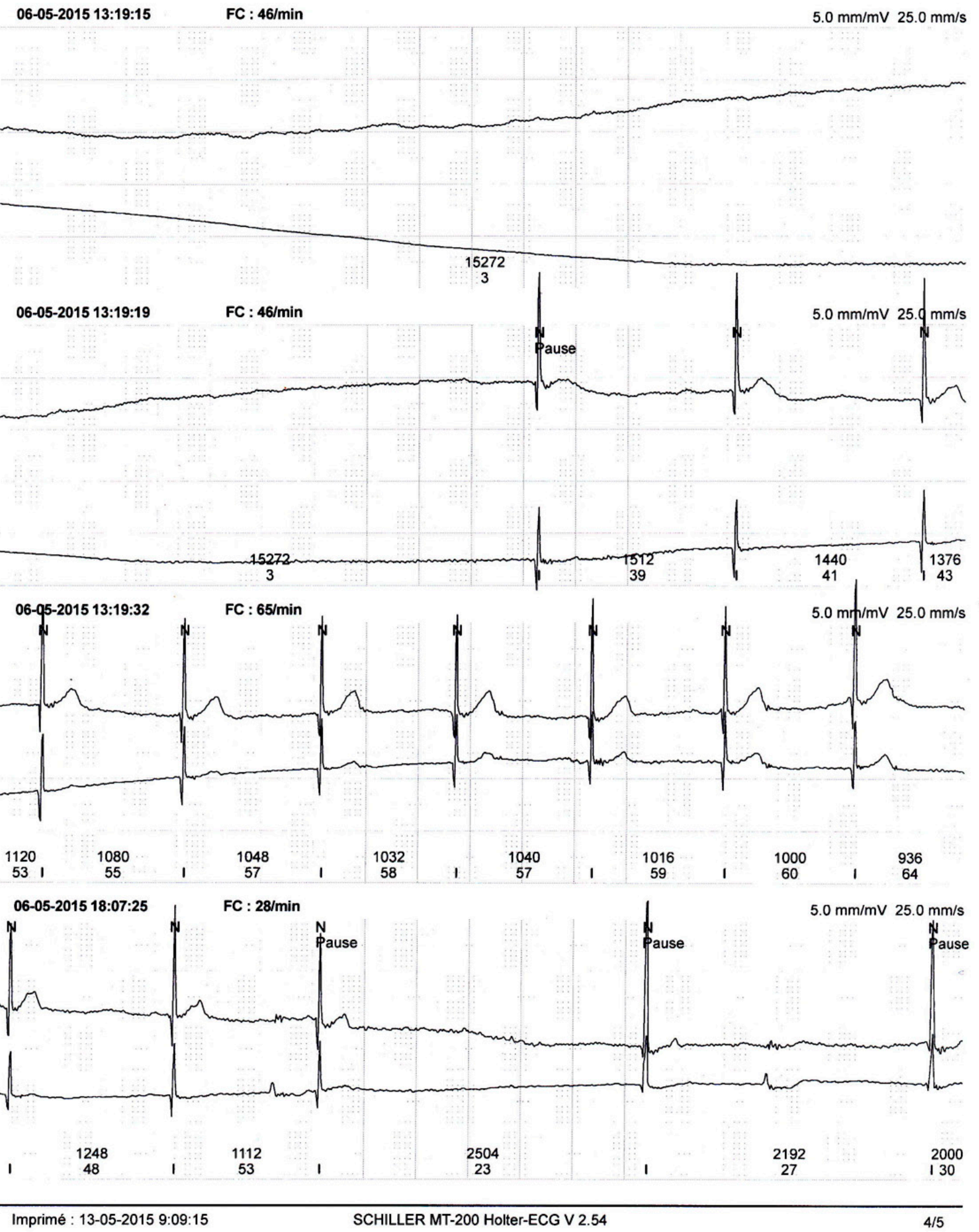


mutations associated with PEPD alter Nav 1.7 by impairing inactivation of the channel leading to an enhanced persistent current. [44] A key unanswered question is why the Nav 1.7 mutations associated with IE and PEPD cause such different phenotypic expression particularly in terms of the distal predominance of symptoms in the former and proximal in the latter [4].

Paroxysmal extreme pain disorder (formerly known as familial rectal pain) is inherited in an autosomal-dominant fashion first described in 1917 by MacLennan. In 1959, rectal, ocular and submaxillary pain (the full spectrum of paroxysmal extreme pain disorder) was described [5]. PEPD is characterized by paroxysmal episodes of severe perineal rectal, ocular, and mandibular pain. The ocular pattern of pain is an intense burning sensation, followed by conjunctival injection and erythema of the eyelids and skin in the temporal region. There are often autonomic manifestations such as skin flushing and bradycardia $[6,7]$. The clinical form for the infant is usually incomplete. The attacks may be precipitated by defecation, crying or yawning. However, symptoms sometimes occurred without any provocative stimulus [8].

Nav 1.7 is found in the dorsal root and sympathetic ganglia. It is particularly highly expressed in nociceptors [9]. The effects of hyper-excitability in these areas correlate to the clinical picture. Pain is associated with autonomic features that can include flushing (which sometimes results in harlequin color change), lacrimation, rhinorrhea, and tonic attacks with apneas and bradycardia. These attacks can escalate to seizures, hypertonia, respiratory arrest, and even asystolic cardiac arrest [6]. Tonic attacks might be confused with epilepsy; however, findings of electroencephalography are normal [10]. Important stimuli are physical factors: defecation or eating but strong emotion can also be a trigger. Between episodes, physical examination is normal. Most patients present symptoms at birth or in infancy. The frequency of rectal pain episodes typically falls with increasing age, although ocular and mandibular pain can become more common. Sudden attacks of severe pain lasting seconds to hours occur, usually in characteristic distributions, namely rectal, ocular, and submaxillary [11].

Diagnosis of these patients requires obtaining a thorough history, examination by a senior neurologist or a pain consultant and referral to an appropriate center for further genetic investigations if necessary, to confirm the diagnostis. Several members of the family might require examination to confirm the genetic diagnosis. Various pharmacological options for providing pain relief have been tried with varying degrees of success including sodium channel blockers, such as carbamazepinr lidocaine mexiletine [12] and central neuraxial local anesthetic blockade [13, 14].

Cardiac syncope must also be considered, especially prolonged QT syndrome, and an ECG is an important first line investigation and, if strongly suspected, 24-hour ECG is recommended. The much rarer channelopathy of paroxysmal extreme pain disorder (PEPD) should also be considered. Miss $S$ is unusual among PEPD patients in that she had had multiple episodes of prolonged pauses. The longest documented asystolic episode has been 15s. These cardiovascular symptoms are not uncommon. The exact etiopathogenesis of seizures and cardiovascular instability is not clearly understood [15]. Although uneventful, we felt that this case was of interest due to the rarity of the condition and the potentially serious adverse outcomes including sudden cardiac arrest.

There are no established guidelines for the management of prolonged sinus pauses for the patient with PEPD. The cardiac stimulation is also indicated. Carbamazepine is effective for reduction of the frequency and severity of attacks in most patients but it did not have a real effect on the reversibility of symptoms in patients with prolonged sinus pauses [12]. 
Perioperative anesthetic considerations include preoperative reassurance and administration of anxiolytics to allay anxiety. There is no established guidance for anesthesia in these patients reported in the anesthetic literature. Because of the potential for cardiovascular instability, it is vital to have resuscitative drugs (vasopressors/inotropes) and invasive monitoring equipment readily available [16]. Although anesthetic technique minimally affects this disorder, regional anesthesia could precipitate the symptoms, possible pressure or pain from needle insertion [17]. If the symptoms appear under general anesthesia, keeping the patient sedated until the disappearance of the symptoms will alleviate any patient discomfort and pain. Provision of adequate perioperative pain relief in these patients can be extremely challenging because of the overexpression of Nav 1.7 receptor in acute pain and inflammatory conditions [17]. Pretreatment with cyclooxygenase 1 and 2 inhibitors could be beneficial in the absence of any contraindications. [10] Multimodal analgesia should be administered to minimize the precipitation of symptoms caused by pain.

\section{Conclusion}

Paroxysmal extreme pain disorder is a highly distinctive sodium channelopathy with incompletely carbamazepine-sensitive bouts of pain and sympathetic nervous system dysfunction. It is most likely to be misdiagnosed as epilepsy and, particularly in infan$c y$, as hyperekplexia and reflex anoxic seizures. The onset of the disorder is in the neonatal period or infancy and persists throughout life. Autonomic manifestations predominate initially, with skin flushing in all and harlequin color change and tonic attacks in most. Dramatic syncopes with bradycardia and sometimes asystole are common. Clinicians must evocate this diagnostic even any clinical suspicion given the severity of cardiac complications.

\section{Consent}

Written informed consent was obtained from the patient for publication of this Case report and any accompanying images. A copy of the written consent is available for review by the Editor of this journal.

\section{List of abbreviations}

-PEPD: Paroxysmal extreme pain disorder.

-VGSCs: voltage-gated sodium channels.

-INaR: Resurgent sodium currents.

-IEM: inherited erythromelalgia.

\section{Competing interests}

The author(s) declare that they have no competing interests.

\section{Authors' contributions}

Sahar Mouram conceived the study and participated in the drafting and development work.

Hicham Sabor and Ibtissam Fellat participated in coordination and helped to draft the manuscript. All authors read and approved the final manuscript. 


\section{References}

1. Estacion M, Dib-Hajj SD, Benke PJ, Rene HM, Morsche TE ,Eastman EM, Macala LJ and al. Nav 1.7 Gain-of-Function Mutations as a Continuum: A1632E Displays Physiological Changes Associated with Erythromelalgia and Paroxysmal Extreme Pain Disorder Mutations and Produces Symptoms of Both Disorders. The Journal of Neuroscience, 22 October 2008, 28(43): 11079-11088.

2. Dib-Hajj SD, Estacion M, Jarecki BW, Tyrrell L, Fischer TZ , Lawden M et al. Paroxysmal extreme pain disorder M1627K mutation in human Nav 1.7 renders DRG neurons hyperexcitable. Molecular Pain 2008, 4(37): 1-14.

3. Theile JW, Brian W, Jarecki AD, Heodore RT. Cummins. Nav 1.7 mutations associated with paroxysmal extreme pain disorder, but not erythromelalgia, enhance Nav $\beta 4$ peptide-mediated resurgent sodium currents. The Journal of Physiology. 589(3): 597-608

4. Caffrey JM , Eng DL, Black JA, Waxman SG, Kocsis JD. Three types of sodium channels in adult rat dorsal root ganglion neurons. Brain Res. 1992; 592(1-2): 283-297.

5. Hayden R , Grossman M. Rectal ocular, and submaxillary pain; a familial autonomic disorder related to proctalgia fugaz: report of a family. AMA J 1959; 97: 479-82

6. Fertleman CR, Ferrie CD, Aicardi J, Bednarek NA, Eeg-Olofsson $\mathrm{O}$, Elmslieand FV et al. Paroxysmal extreme pain disorder (previously familial rectal pain syndrome). Neurology 2007; 69: 586-95.

7. Fertleman $C R$, Baker MD, Parker KA, et al. SCN9A mutations in paroxysmal extreme pain disorder: allelic variants underlie distinct channel defects and phenotypes. Neuron 2006; 52: 767-74.

8. Choi JS, Waxman SG. Physiological interactions between Nav 1.7 and Nav 1.8 sodium channels: a computer simulation study. J Neurophysiol 2011; 106: 3173-84.

9. Lavin MM. , Solano C. Dorsal root ganglia, sodium channels, and fibromyalgia sympathetic pain. Medical Hypotheses. 2009; 72: 64-66.

10. Emery EC , Habib AM ,Cox JJ , Nicholas AK, Gribble FM ,Woods CG ,Reimann F. Novel SCN9A Mutations Underlying Extreme Pain Phenotypes: Unexpected Electrophysiological and Clinical Phenotype Correlations. The Journal of Neuroscience.2015; 35(20): 7674-7681.

11. Dugan RE. Familial rectal pain. Lancet 1972; 1: 854;

12. Black JA, Dibb-Hajj S, McNabola K, Jeste S, Rizzo MA, Kocsis JD, Waxman SG. Spinal sensory neurons express multiple sodium channel alpha subunit mRNAs. Mol Brain Res 1996;43: 117-31;

13. Harrison CM, Goddard JM, Rittey CD. The use of regional anaesthetic blockade in a child with recurrent erythromelalgia. Arch Dis Child 2003;88: 65-6.
14. Brar HBK, El-Dabe S, Shehade S. Treatment of refractory primary erythromelalgia in a child using a continuous epidural infusion. Pain Clin 2002;12: 65-9.

15. Nassar MA, Levato A, Stirling LC, Wood JN. Neuropathic pain develops normally in mice lacking both Nav 1.7 and Nav 1.8. Mol Pain 2005;1: 24.

16. Darbar A ,Bilolikar A.Anesthesia for a Patient with Paroxysmal Extreme Pain Disorder.

17. Gould HJ III, Engl JD, Soignier RD, Nolan P, Minor LD, Liu ZP et al. Ibuprofen blocks changes in Nav 1.7 and 1.8 sodium channels associated with complete Freund's adjuvant-induced inflammation in rat. Pain 2004;5: 270-80.

\section{Comment on this article:}

\section{(f) 8 in $8+\mathbf{S} P$}

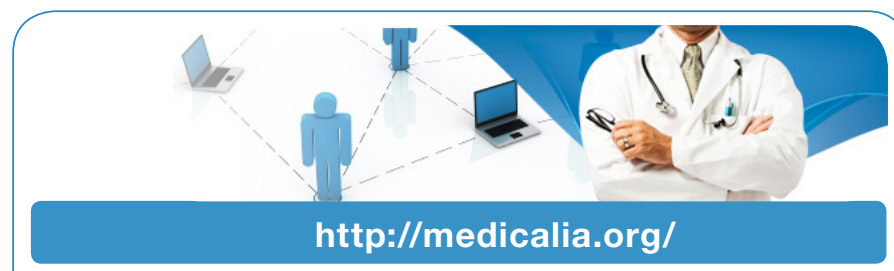

Where Doctors exchange clinical experiences, review their cases and share clinical knowledge. You can also access lots of medical publications for free. Join Now!

\section{Publish with iMedPub}

http://www.imed.pub

International Archives of Medicine is an open access journal publishing articles encompassing all aspects of medical science and clinical practice. IAM is considered a megajournal with independent sections on all areas of medicine. IAM is a really international journal with authors and board members from all around the world. The journal is widely indexed and classified Q1 in category Medicine. 